\title{
Thermal radiation effect on an unsteady MHD free convective chemically reacting viscous dissipative fluid flow past an infinite vertical moving porous plate with Heat source
}

\author{
Mamta Thakur \& M. Venkata Krishna \\ Department of Mathematics, University College of Science, Osmania University, Hyderabad, 500007, Andhra \\ Pradesh, India.
}

\begin{abstract}
Numerical solutions for heat and mass transfer by laminar flow of a Newtonian, viscous, electrically conducting chemically reacting viscous dissipative fluid on a continuously vertical permeable surface in the presence of a heat source, a first - order homogeneous chemical reaction and the mass flux are reported. The plate is assumed to move with a constant velocity in the direction of fluid flow. A uniform magnetic field acts perpendicular to the porous surface, which absorbs the fluid with a suction velocity varying with time. The fluid is considered to be a gray, absorbing emitting but non - scattering medium and the Rosseland approximation is used to describe the radiative heat flux in the energy equation. The problem is governed by coupled non - linear partial differential equations. Dimensionless equations of the problem have been solved numerically by finite element method. The effects of flow parameters, viz. thermal Grashof number, solutal Grashof number, Hartmann number, Permeability parameter, Thermal radiation parameter, Prandtl number, Heat source parameter, Schmidt number and Eckert number on velocity, temperature and concentration fields are investigated through graphs. Skin friction, Rate of heat and mass transfer coefficients are derived, discussed numerically and presented in tabular forms. The combined heat and mass transfer plays an important role are designing chemical processing equipment, formation and dispersion of fog, distribution of temperature and moisture over agricultural fields and groves of fruit trees, crop damage due to freezing, and environmental pollution.
\end{abstract}

Keywords: MHD, Free convection, Viscous dissipation, Porous medium, Thermal radiation, Chemical reaction and Finite element method.

\begin{tabular}{|c|c|c|c|}
\hline \multicolumn{4}{|c|}{ Nomenclature: } \\
\hline$A$ & A constant & $T_{\infty}^{\prime}$ & Temperature of the fluid at infinity \\
\hline$B_{o}$ & Magnetic field component along $y^{\prime}-$ & $C$ & Concentration of the fluid \\
\hline & & $C^{\prime}$ & Concentration of fluid near the plate \\
\hline$C_{p}$ & Specific heat at constant pressure & $C_{w}^{\prime}$ & Concentration of the fluid far away of the \\
\hline$G r$ & thermal Grash of number & & fluid from the plate \\
\hline Gc & solutalGrashof number & $C_{\infty}^{\prime}$ & Concentration of the fluid at infinity \\
\hline$g$ & Acceleration of gravity & $t^{\prime}$ & Time in $x^{\prime}, y^{\prime}$ coordinate system \\
\hline$K^{\prime}$ & The permeability of medium & $t$ & Time in dimensionless co - ordinates \\
\hline $\begin{array}{l}K \\
M\end{array}$ & $\begin{array}{l}\text { 1 he permeability parameter } \\
\text { Hartmann number }\end{array}$ & $u^{\prime}$ & Velocity component in $x^{\prime}-$ direction \\
\hline $\operatorname{Pr}$ & Prandtl number & $u$ & Dimensionless velocity component in \\
\hline$S c$ & Schmidt number & $\mathrm{Nu}$ & $\begin{array}{l}x-\text { direction } \\
\text { Nusselt number }\end{array}$ \\
\hline Ec & Eckert number & $S h$ & Sherwood number \\
\hline $\mathrm{S}$ & Heat source parameter & $R_{e}$ & Reynold's number \\
\hline$R$ & Thermal radiation parameter & $\boldsymbol{n}_{e_{x}}$ & \\
\hline$U_{p}$ & Plate Velocity & $\begin{array}{l}\bar{K} \\
\text { with }\end{array}$ & $\begin{array}{l}\text { Chemical reaction of first order } \\
\text { rate constant }\end{array}$ \\
\hline$k_{r}$ & Chemical reaction parameter & $x^{\prime}, y^{\prime}$ & $\mathrm{Co}-$ ordinate system \\
\hline$D$ & Chemical molecular diffusivity & $x, y$ & Dimension lesscoordinates \\
\hline$T^{\prime}$ & Temperature of fluid near the plate & $U_{o}$ & Reference velocity \\
\hline & fluid from the plate & $V_{o}$ & Suction velocity \\
\hline
\end{tabular}




\author{
Greek symbols: \\ $\beta$ Coefficient of volume expansion \\ for heat transfer \\ $\beta^{*} \quad$ Coefficient of volume expansion for mass \\ transfer \\ $\kappa \quad$ Thermal conductivity of the fluid \\ $\sigma \quad$ Electrical conductivity of the fluid \\ $v \quad$ Kinematic viscosity
}

$\begin{array}{ll}\theta & \text { Non - dimensional temperature } \\ \rho & \text { Density of the fluid } \\ \alpha & \text { Angle } \\ n t & \text { Phase angle } \\ \varepsilon & \text { A positive constant } \\ \tau & \text { Skin - friction }\end{array}$

\section{Introduction:}

Combined heat and mass transfer problems with chemical reaction are of importance in many processes and have, therefore, received a considerable amount of attention in recent years. In processes such as drying, evaporation at the surface of a water body, energy transfer in a wet cooling tower and the flow in a desert cooler, heat and mass transfer occur simultaneously. Possible applications of this type of flow can be found in many industries. For example, in the power industry, among the methods of generating electric power is one in which electrical energy is extracted directly from a moving conducting fluid. We are particularly interested in cases in which diffusion and chemical reaction occur at roughly the same speed. When diffusion is much faster than chemical reaction, then only chemical factors influence the chemical reaction rate; when diffusion is not much faster than reaction, the diffusion and kinetics interact to produce very different effects. The study of heat generation or absorption effects in moving fluids is important in view of several physical problems, such as fluids undergoing exothermic or endothermic chemical reaction. Due to the fast growth of electronic technology, effective cooling of electronic equipment has become warranted and cooling of electronic equipment ranges from individual transistors to main frame computers and from energy suppliers to telephone switch boards and thermal diffusion effect has been utilized for isotopes separation in the mixture between gases with very light molecular weight (hydrogen and helium) and medium molecular weight.

The Effects of Variable properties and Hall current on steady MHD laminar convective fluid flow due to a porous rotating disk was studied by Abdur Sattar et al. [1]. Anjali Devi and Wilfred Samuel Raj [2] investigated the effects of thermo diffusion on unsteady hydromagnetic free convection flow of a viscous, incompressible, electrically conducting fluid with heat and mass transfer past a moving porous vertical plate of infinite length with time dependent suction in the presence of heat source in a slip flow regime. Slip flow conditions for the velocity and jump in temperature are taken into account in the boundary conditions. A uniform transverse magnetic field is applied. The free stream velocity is considered to follow an exponentially small perturbation law. The governing equations of the problem subject to the slip flow boundary conditions are solved analytically using the perturbation method.Dulal Pal et al. [3]studied Perturbation analysis of unsteady magnetohydrodynamic convective heat and mass transfer in a boundary layer slip flow past a vertical permeable plate with thermal radiation and chemical reaction. Ibrahim et al. [4] studied the effects of chemical reaction and radiation absorption on transient hydromagnetic natural convection flow with wall transpiration and heat source. Analytical solutions for heat and mass transfer by laminar flow of a Newtonian, viscous, electrically conducting and heat generation/ absorbing fluid on a continuously vertical permeable surface in the presence of a radiation, a first - order homogeneous chemical reaction and the mass flux are reported by Kesavaiah et al. [5]. The plate is assumed to move with a constant velocity in the direction of fluid flow. A uniform magnetic field acts perpendicular to the porous surface, which absorbs the fluid with a suction velocity varying with time. The dimensionless governing equations for this investigation are solved analytically using two - term harmonic and non - harmonic functions. Finite difference solution of the homogeneous first order chemical reaction on unsteady flow past an impulsively started semi - infinite vertical plate with variable temperature and massdiffusion in the presence of thermal radiation have been studied by Loganathan et al. [6]. The fluid considered here is a gray, absorbing - emitting radiation but a non - scattering medium. The dimensionless governing equations are solved by an efficient, more accurate and unconditionally stable and fast converging implicit scheme. Mansour et al.[7] investigates the influence of chemical reaction and viscous dissipation on magnetohydrodynamic natural convection flow. An approximate numerical solution for the flow problem has been obtained by solving the governing equations using shooting technique with a fourth - order Runge - Kutta integration scheme. Four different cases of flows have been studied namely an isothermal surface, a uniform heat flux surface, a plane plume and flow generated from a horizontal line energy source a vertical adiabatic surface. Double - Diffusive Convection - Radiation interaction on unsteady MHD flow over a vertical moving porous plate with heat generation and Soret effects was studied by Mohamed [8]. Free convection flow of a viscous incompressible flow past an oscillating infinite vertical plate with variable temperature and mass diffusion has been studied by Muthucumaraswamy and Vijayalakshmi [9]. In this research paper, the dimensionless governing equations are tackled using Laplace transform technique and the temperature and 
species concentration near the plate are assumed to be linearly varying with respect to time. Muthucumaraswamy and Meenakshisundaram [10] investigated theoretical study of chemical reaction effects on vertical oscillating plate with variable temperature and mass diffusion. There has been a renewed interest in studying magnetohydrodynamic (MHD) flow and heat transfer in porous and non-porous media due to the effect of magnetic fields on the boundary layer flow control and on the performance of many systems using electrically conducting fluids. Muthuraj and Srinivas [11] studied the fully developed MHD flow of a micropolar and viscous fluid in a vertical porous space using HAM.Unsteady MHD free Convective flow of a compressible fluid past a moving vertical plate in the presence of Radiative heat transfer was studied by Ogulu et al. [12].

The study of heat generation or absorption effects in moving fluids is important in view of several physical problems, such as fluids undergoing exothermic or endothermic chemical reactions. Possible heat generation effects may alter the temperature distribution and consequently, the particle deposition rate in nuclear reactors, electric chips and semiconductor wafers. Patil and Kulkarni [13] studied the effects of chemical reaction on free convective flow of a polar fluid through porous medium in the presence of internal heat generation. Radiation effects on an unsteady MHD convective heat and mass transfer flow past a semi - infinite vertical permeable moving plate embedded in a porous medium was studied by Ramachandra Prasad et al. [14]. Ramana Reddy et al. [15] have studied the mass transfer and radiation effects of unsteady MHD free convective fluid flow embedded in porous medium with heat generation/absorption. In spite of all these studies, the unsteady MHD free convection heat and mass transfer flow past a moving semi - infinite titled porous plate with variable temperature in the presence of chemical reaction has received a little attention. MHD boundary layer flow over a heated stretching sheet with variable Viscosity was studied by Samad et al. [16]. Satyanarayana and Venkataramana [17] studied Hall current effect on magnetohydrodynamics free convection flow past a semi - infinite vertical porous plate with mass transfer. Seddeek [18] studied the effects of chemical reaction, thermophoresis and variable viscosity on steady hydromagnetic flow with heat and mass transfer over a flat plate in the presence of heat generation/absorption. Sharma and Singh [19] investigates the flow of a viscous incompressible electrically conducting fluid along a porous vertical isothermal non - conducting plate with variable suction and exponentially decaying heat generation in the presence of transverse magnetic field. The governing equations of motion and energy are transformed into ordinary differential equations using time dependent similarity parameter. The ordinary differential equations are then solved numerically using Runge Kutta method along with shooting technique. Effects of the chemical reaction and radiation absorption on free convection flow through porous medium with variable suction in the presence of uniform magnetic field were studied by Sudheer Babu and Satyanarayana [20]. In spite of all these studies, the unsteady MHD free convection heat and mass transfer for a heat generating fluid with radiation absorption has received little attention. Venkateswalu et al. [21] deal with free convention flow along a vertical wall in a porous medium with magnetic field is considered. The horizontal walls are adiabatic. The magnetic field applied perpendicular to the rectangular channel. The flow problem axis described by means of parabolic partial differential equations and solutions are obtained by an implicit finite difference technique. Zueco [22] considered the transient magnetohydrodynamic natural convection boundary layer flow with suction, viscous dissipation and thermal radiation effects.

The effect of radiation on MHD flow and heat transfer problem has become more important industrially. At high operating temperature, radiation effect can be quite significant. Many processes in engineering areas occur at high temperature and knowledge of radiation heat transfer becomes very important for design of reliable equipment, nuclear plants, gas turbines and various propulsion devices or aircraft, missiles, satellites and space vehicles. Based on these applications, Cogley et al. [23] showed that in the optically thin limit, the fluid does not absorb its own emitted radiation but the fluid does absorb radiation emitted by the boundaries. Satter and Hamid [24] investigated the unsteady free convection interaction with thermal radiation in a boundary layer flow past a vertical porous plate. Vajravelu [25] studied the flow of a steady viscous fluid and heat transfer characteristic in a porous medium by considering different heating processes. Hossain and Takhar [26] have considered the radiation effect on mixed convection boundary layer flow of an optically dense viscous incompressible fluid along a vertical plate with uniform surface temperature. Raptis [27] investigate the steady flow of a viscous fluid through a porous medium bounded by a porous plate subjected to a constant suction velocity by the presence of thermal radiation. Makinde [28] examined the transient free convection interaction with thermal radiation of an absorbing emitting fluid along moving vertical permeable plate. The effect of the chemical reaction and radiation absorption on the unsteady MHD free convection flow past a semi - infinite vertical permeable moving plate with heat source and suction has been studied by Ibrahim et al. [29]. Recently Bakr [30] presented an analysis on MHD free convection and mass transfer adjacent to moving vertical plate for micropolar fluid in a rotating frame of reference in presence of heat generation/ absorption and a chemical reaction. 
Hence, the main objective of the present investigation is to study the effects of radiation absorption, mass diffusion, chemical reaction and heat source parameter of heat generating fluid past a vertical porous plate subjected to variable suction. It is assumed that the plate is embedded in a uniform porous medium and moves with a constant velocity in the flow direction in the presence of a transverse magnetic field. It is also assumed that temperature over which are superimposed an exponentially varying with time. The equations of continuity, linear momentum, energy and diffusion, which govern the flow field, are solved numerically by using finite element method which is more economical from computational view point and the results obtained are good agreement with the results of and the results obtained are good agreement with the results of Kesavaiah et al. [5] in some special cases. The behavior of the velocity, temperature, concentration, skin - friction, Nusselt number and Sherwood number has been discussed for variations in the governing parameters.

\section{Mathematical formulation:}

We consider unsteadytwodimensional flow of a laminar, viscous, electrically conducting chemically reacting viscous dissipative and heatabsorbing fluid past a semiinfinite vertical permeable moving plate embedded in a uniform porous medium and subjected to a uniform transverse magnetic field in the presence of thermal and concentration buoyancy effects.

We made the following assumptions.

1. In Cartesian coordinate system, let $x^{\prime}$ - axis is taken to be along the plate and the $y^{\prime}$-axis normal to the plate. Since the plate is considered infinite in $x^{\prime}$-direction, hence all physical quantities will be independent of $x^{\prime}$ - direction.

2. The wall is maintained at constant temperature $\left(T_{w}^{\prime}\right)$ and concentration $\left(C_{w}^{\prime}\right)$ higher than the ambient temperature $\left(T_{\infty}^{\prime}\right)$ and concentration $\left(C_{\infty}^{\prime}\right)$ respectively.

3. A uniform magnetic field of magnitude $B_{o}$ is applied normal to the plate. The transverse applied magnetic field and magnetic Reynold's number are assumed to be very small, so that the induced magnetic field is negligible.

4. A uniform magnetic field acts perpendicular to the porous surface, which absorbs the fluid with a suction velocity varying with time.

5. The plate is assumed to move with a constant velocity in the direction of fluid flow.

6. The fluid properties are assumed to be constant except that the influence of density variation with temperature has been considered only in the body - force term.

7. The chemical reactions are taking place in the flow and all thermophysical properties are assumed to be constant of the linear momentum equation which is approximated according to the Boussinesq's approximation.

8. Due to the semi - infinite plane surface assumption, the flow variables are functions of $y^{\prime}$ and the time $t^{\prime}$ only.

9. It is assumed that there is no applied voltage which implies the absence of an electric field. The fluid has constant kinematic viscosity and constant thermal conductivity, and the Boussinesq's approximation have been adopted for the flow. 


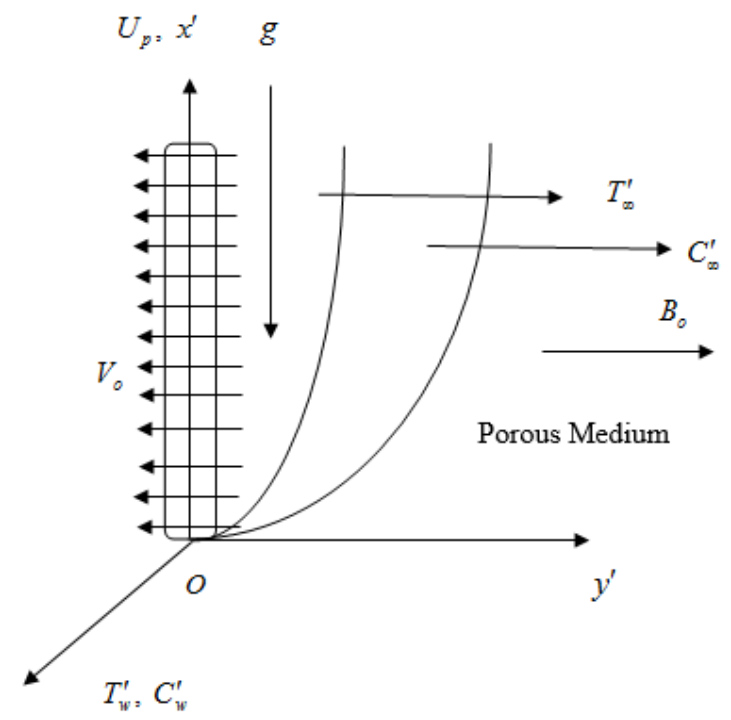

Figure 1: Physical model and coordinate system of the problem

10. The fluid is considered to be gray absorbing - emitting radiation but non - scattering medium and the Rosseland's approximation is used to describe the radiative heat flux. It is considered to be negligible in $x^{\prime}-$ direction as compared in $y^{\prime}-$ direction.

11. At time $t^{\prime}>0$ the plate is given an impulsive motion in the direction of flow i.e. along $x^{\prime}$ - axis against the gravity with constant velocity $U_{o}$.In addition, it is assumed that the temperature and the concentration at the wall as well as the suction velocity are exponentially varying with time.

12. The concentration of diffusing species is very small in comparison to other chemical species, the concentration of species far from the wall, $C_{\infty}^{\prime}$, is infinitesimally small and hence the Soret and Dufour effects are neglected.

Under these assumptions, the equations that describe the physical situation are given by Equation of Continuity:

$\frac{\partial v^{\prime}}{\partial y^{\prime}}=0(1)$

Momentum Equation:

$\frac{\partial u^{\prime}}{\partial t^{\prime}}+v^{\prime} \frac{\partial u^{\prime}}{\partial y^{\prime}}=v \frac{\partial^{2} u^{\prime}}{\partial y^{\prime 2}}+g \beta\left(T^{\prime}-T_{\infty}^{\prime}\right)+g \beta^{*}\left(C^{\prime}-C_{\infty}^{\prime}\right)-\left(\frac{\sigma B_{o}^{2}}{\rho}+\frac{v}{K^{\prime}}\right) u^{\prime}(2)$

Energy Equation:

$\frac{\partial T^{\prime}}{\partial t^{\prime}}+v^{\prime} \frac{\partial T^{\prime}}{\partial y^{\prime}}=\frac{1}{\rho C_{p}} \kappa \frac{\partial^{2} T^{\prime}}{\partial y^{\prime 2}}+S^{\prime}\left(T^{\prime}-T_{\infty}^{\prime}\right)+\frac{v}{C_{p}}\left(\frac{\partial u^{\prime}}{\partial y^{\prime}}\right)^{2}-\frac{\partial q_{r}}{\partial y^{\prime}}(3)$

Species Diffusion Equation:

$\frac{\partial C^{\prime}}{\partial t^{\prime}}+v^{\prime} \frac{\partial C^{\prime}}{\partial y^{\prime}}=D \frac{\partial^{2} C^{\prime}}{\partial y^{\prime 2}}-K_{r}^{\prime}\left(C^{\prime}-C_{\infty}^{\prime}\right)$

13. The third and fourth terms on the right hand side of equation (2) denote the thermal and concentration buoyancy effects respectively. Also the last term on the right hand side of the energy equation (3) represents the radiative heat flux term.

With the following initial and boundary conditions:

$$
\begin{aligned}
& t^{\prime} \leq 0: u^{\prime}=0, T^{\prime}=T_{\infty}^{\prime}, C^{\prime}=C_{\infty}^{\prime} \text { for all } y^{\prime} \\
& t^{\prime}>0:\left\{\begin{array}{l}
u^{\prime}=u_{p}^{\prime}, T^{\prime}=T_{\infty}^{\prime}+\varepsilon\left(T_{w}^{\prime}-T_{\infty}^{\prime}\right) e^{n^{\prime} t^{\prime}}, C^{\prime}=C_{\infty}^{\prime}+\varepsilon\left(C_{w}^{\prime}-C_{\infty}^{\prime}\right) e^{n^{\prime} t^{\prime}} \text { at } y^{\prime}=0 \\
u^{\prime} \rightarrow 0, T^{\prime} \rightarrow 0, C^{\prime} \rightarrow 0 \text { as } y^{\prime} \rightarrow \infty
\end{array}\right\}
\end{aligned}
$$


From equation (1), it is clear that the suction velocity at the plate is either a constant or a function of time. Hence the suction velocity normal to the plate is assumed in the form

$v^{\prime}=-V_{o}\left(1+\varepsilon A e^{n^{\prime} t^{\prime}}\right)$

(6)

Where $A$ is a real positive constant, $\varepsilon$ and $\varepsilon A$ is small values less than unity, and $V_{o}$ is scale of suction velocity which is non - zero positive constant. The negative sign indicates that the suction is towards the plate.

The radiative heat flux term is simplified by making use of the Rosseland approximation [31] as $q_{r}=-\frac{4 \bar{\sigma}}{3 k^{*}} \frac{\partial T^{\prime 4}}{\partial y^{\prime}}$

Here $\bar{\sigma}$ is Stefan - Boltzmann constant and $k^{*}$ is the mean absorption coefficient. It is assumed that the temperature differences within the flow are sufficiently small so that $T^{\prime 4}$ can be expressed as a linear function of $T^{\prime}$ after using Taylor's series to expand $T^{\prime 4}$ about the free stream temperature $T_{h}^{\prime}$ and neglecting higher order terms. This results in the following approximation:

$T^{\prime 4} \cong 4 T_{h}^{\prime 3} T^{\prime}-3 T_{h}^{\prime 4}$

Using equations (7) and (8) in the last term of equation (3), we obtain:

$\frac{\partial q_{r}}{\partial y}=-\frac{16 \bar{\sigma} T_{h}^{\prime 3}}{3 k^{*}} \frac{\partial^{2} T^{\prime}}{\partial y^{\prime 2}}(9)$

Introducing (9) in the equation (3), the energy equation becomes:

$\frac{\partial T^{\prime}}{\partial t^{\prime}}+v^{\prime} \frac{\partial T^{\prime}}{\partial y^{\prime}}=\frac{\kappa}{\rho C_{p}} \frac{\partial^{2} T^{\prime}}{\partial y^{\prime 2}}+\frac{v}{C_{p}}\left(\frac{\partial u^{\prime}}{\partial y^{\prime}}\right)^{2}+S^{\prime}\left(T^{\prime}-T_{\infty}^{\prime}\right)+\frac{16 \bar{\sigma} T_{h}^{\prime 3}}{3 k^{*}} \frac{\partial^{2} T^{\prime}}{\partial y^{\prime 2}}(10)$

In order to write the governing equations and the boundary conditions in dimensionless from, the following non - dimensional quantities are introduced.

$$
\begin{aligned}
& \left.\begin{array}{l}
y=\frac{y^{\prime} V_{o}}{v}, t=\frac{t^{\prime} V_{o}^{2}}{v}, u=\frac{u^{\prime}}{U_{o}}, v=\frac{v^{\prime}}{V_{o}^{2}}, \theta=\frac{T^{\prime}-T_{\infty}^{\prime}}{T_{w}^{\prime}-T_{\infty}^{\prime}}, C=\frac{C^{\prime}-C_{\infty}^{\prime}}{C_{w}^{\prime}-C_{\infty}^{\prime}}, n=\frac{u^{\prime} v}{V_{o}^{2}}, \\
U_{p}=\frac{u_{p}^{\prime}}{U_{o}}, G r=\frac{g \beta v\left(T_{w}^{\prime}-T_{\infty}^{\prime}\right)}{U_{o} V_{o}^{2}}, G c=\frac{g \beta^{*} v\left(C_{w}^{\prime}-C_{\infty}^{\prime}\right)}{U_{o} V_{o}^{2}}, \operatorname{Pr}=\frac{v \rho C_{p}}{\kappa}=\frac{v}{\alpha}, S c=\frac{v}{D}, \\
M=\left(\frac{\sigma B_{o}^{2}}{\rho}\right) \frac{v}{V_{o}^{2}}, S=\frac{v Q_{o}}{\rho C_{p} V_{o}^{2}}, K=\frac{K^{\prime} V_{o}^{2}}{v^{2}}, k_{r}=\frac{K_{r}^{\prime} v}{V_{o}^{2}}, R=\frac{\kappa k^{*}}{4 \sigma T_{h}^{3}}, E c=\frac{V_{o}^{2}}{C_{p}\left(T_{w}^{\prime}-T_{\infty}^{\prime}\right)}
\end{array}\right\}
\end{aligned}
$$

Substituting the above non - dimensional quantities (11) in the equations (2), (4) and (10) then these equations are reducing to the following dimensional form:

$$
\begin{aligned}
& \frac{\partial u}{\partial t}-\left(1+\varepsilon A e^{n t}\right) \frac{\partial u}{\partial y}=\frac{\partial^{2} u}{\partial y^{2}}+\left(M+\frac{1}{K}\right) u+(G r) \theta+(G c) C \\
& \frac{\partial \theta}{\partial t}-\left(1+\varepsilon A e^{n t}\right) \frac{\partial \theta}{\partial y}=\frac{1}{\operatorname{Pr}}\left(1+\frac{4}{3 R}\right) \frac{\partial^{2} \theta}{\partial y^{2}}+S \theta+(E c)\left(\frac{\partial u}{\partial y}\right)^{2} \\
& \frac{\partial C}{\partial t}-\left(1+\varepsilon A e^{n t}\right) \frac{\partial C}{\partial y}=\frac{1}{S c} \frac{\partial^{2} C}{\partial y^{2}}-\left(k_{r}\right) C(14)
\end{aligned}
$$

The corresponding initial and boundary conditions in dimensionless form are:

$$
\begin{aligned}
& t \leq 0: u=0, \theta=0, C=0 \text { for all } y \\
& t>0:\left\{\begin{array}{l}
u=U_{p}, \theta=1+\varepsilon e^{n t}, C=1+\varepsilon e^{n t} \text { at } y=0 \\
u \rightarrow 0, \theta \rightarrow 0, C \rightarrow 0 \text { as } y \rightarrow \infty
\end{array}\right\} \text { (15) }
\end{aligned}
$$


All the physical parameters are defined in the nomenclature.

It is now important to calculate the physical quantities of primary interest, which are the local wall shear stress, the local surface heat and mass flux. Given the velocity field in the boundary layer, we can now calculate the local wall shear stress (i.e., skin - friction) is given by and in dimensionless form, we obtain knowing the temperature field, it is interesting to study the effect of the free convection and radiation on the rate of heat transfer. This is given by which is written in dimensionless form as

$$
\tau=\frac{\tau_{w}}{\rho u_{w}^{2}}, \tau_{w}=\left[\mu \frac{\partial u}{\partial y}\right]_{y^{\prime}=0}=\rho V_{o}^{2} u^{\prime}(0)=\left[\frac{\partial u}{\partial y}\right]_{y=0}
$$

The dimensionless local surface heat flux (i.e., Nusselt number) is obtained as

$$
N_{u}\left(x^{\prime}\right)=-\left[\frac{x^{\prime}}{\left(T_{w}^{\prime}-T_{\infty}^{\prime}\right)} \frac{\partial T^{\prime}}{\partial y^{\prime}}\right]_{y^{\prime}=0} \text { then } N u=\frac{N_{u}\left(x^{\prime}\right)}{R_{e_{x}}}=-\left[\frac{\partial \theta}{\partial y}\right]_{y=0}
$$

The definition of the local mass flux and the local Sherwood number are respectively given bywith the help of these equations, one can write

$$
S_{h}\left(x^{\prime}\right)=-\left[\frac{x^{\prime}}{\left(C_{w}^{\prime}-C_{\infty}^{\prime}\right)} \frac{\partial C^{\prime}}{\partial y^{\prime}}\right]_{y^{\prime}=0} \text { then } S h=\frac{S_{h}\left(x^{\prime}\right)}{R_{e_{x}}}=-\left[\frac{\partial C}{\partial y}\right]_{y=0}
$$

Where $R_{e_{x}}=-\frac{V_{o} x^{\prime}}{v}$ is the Reynold's number.

\section{Method of Solution:}

By applying Galerkin finite element method for equation (12) over the element $(e),\left(y_{j} \leq y \leq y_{k}\right)$ is:

$$
\int_{y_{j}}^{y_{k}}\left\{N^{T}\left[\frac{\partial^{2} u^{(e)}}{\partial y^{2}}-\frac{\partial u^{(e)}}{\partial t}+B \frac{\partial u^{(e)}}{\partial y}-Y u^{(e)}+P\right]\right\} d y=0
$$

Where $B=1+\varepsilon A e^{n t}, Y=M+\frac{1}{K}, P=(G r) \theta+(G c) C$

Integrating the first term in equation (19) by parts one obtains

$$
N^{(e)^{T}}\left\{\frac{\partial u^{(e)}}{\partial y}\right\}_{y_{j}}^{y_{k}}-\int_{y_{j}}^{y_{k}}\left\{\frac{\partial N^{(e)^{T}}}{\partial y} \frac{\partial u^{(e)}}{\partial y}+N^{(e)^{T}}\left(\frac{\partial u^{(e)}}{\partial t}-B \frac{\partial u^{(e)}}{\partial y}+Y u^{(e)}-P\right)\right\} d y=0
$$

Neglecting the first term in equation (20), one gets:

$$
\int_{y_{j}}^{y_{k}}\left\{\frac{\partial N^{(e)^{T}}}{\partial y} \frac{\partial u^{(e)}}{\partial y}+N^{(e)^{T}}\left(\frac{\partial u^{(e)}}{\partial t}-B \frac{\partial u^{(e)}}{\partial y}+Y u^{(e)}-P\right)\right\} d y=0
$$

Let $u^{(e)}=N^{(e)} \phi^{(e)}$ be the linear piecewise approximation solution over the element $(e)$

$\left.y_{j} \leq y \leq y_{k}\right)$, where $N^{(e)}=\left[\begin{array}{ll}N_{j} & N_{k}\end{array}\right], \phi^{(e)}=\left[\begin{array}{ll}u_{j} & u_{k}\end{array}\right]^{T}$ and $\quad N_{j}=\frac{y_{k}-y}{y_{k}-y_{j}}, N_{k}=\frac{y-y_{j}}{y_{k}-y_{j}}$ are

the basis functions. One obtains:

$$
\begin{aligned}
& \int_{y_{j}}^{y_{k}}\left\{\left[\begin{array}{cc}
N_{j}^{\prime} N_{j}^{\prime} & N_{j}^{\prime} N_{k}^{\prime} \\
N_{j}^{\prime} N_{k}^{\prime} & N_{k}^{\prime} N_{k}^{\prime}
\end{array}\right]\left[\begin{array}{l}
u_{j} \\
u_{k}
\end{array}\right]\right\} d y+\int_{y_{j}}^{y_{k}}\left\{\left[\begin{array}{cc}
N_{j} N_{j} & N_{j} N_{k} \\
N_{j} N_{k} & N_{k} N_{k}
\end{array}\right]\left[\begin{array}{c}
\dot{u_{j}} \\
\dot{u_{k}}
\end{array}\right]\right\} d y-B \int_{y_{j}}^{y_{k}}\left\{\left[\begin{array}{cc}
N_{j} N_{j}^{\prime} & N_{j} N_{k}^{\prime} \\
N_{j}^{\prime} N_{k} & N_{k}^{\prime} N_{k}
\end{array}\right]\left[\begin{array}{l}
u_{j} \\
u_{k}
\end{array}\right]\right\} d y \\
& +Y \int_{y_{j}}^{y_{k}}\left\{\left[\begin{array}{ccc}
N_{j} N_{j} & N_{j} N_{k} \\
N_{j} N_{k} & N_{k} N_{k}
\end{array}\right]\left[\begin{array}{l}
u_{j} \\
u_{k}
\end{array}\right]\right\} d y=P \int_{y_{j}}^{y_{k}}\left[\begin{array}{c}
N_{j} \\
N_{k}
\end{array}\right] d y
\end{aligned}
$$

Simplifying we get 


$$
\frac{1}{l^{(e)^{2}}}\left[\begin{array}{rr}
1 & -1 \\
-1 & 1
\end{array}\right]\left[\begin{array}{l}
u_{j} \\
u_{k}
\end{array}\right]+\frac{1}{6}\left[\begin{array}{ll}
2 & 1 \\
1 & 2
\end{array}\right]\left[\begin{array}{l}
\dot{u}_{j} \\
\dot{u}_{k}
\end{array}\right]-\frac{B}{2 l^{(e)}}\left[\begin{array}{ll}
-1 & 1 \\
-1 & 1
\end{array}\right]\left[\begin{array}{l}
u_{j} \\
u_{k}
\end{array}\right]+\frac{Y}{6}\left[\begin{array}{ll}
2 & 1 \\
1 & 2
\end{array}\right]\left[\begin{array}{l}
u_{j} \\
u_{k}
\end{array}\right]=\frac{P}{2}\left[\begin{array}{l}
1 \\
1
\end{array}\right]
$$

Where prime and dot denotes differentiation w. r. $\mathrm{t} y$ and time $t$ respectively. Assembling the element equations for two consecutive elements $y_{i-1} \leq y \leq y_{i}$ and $y_{i} \leq y \leq y_{i+1}$ following is obtained:

$$
\frac{1}{l^{(e)^{2}}}\left[\begin{array}{rrr}
1 & -1 & 0 \\
-1 & 2 & -1 \\
0 & -1 & 1
\end{array}\right]\left[\begin{array}{l}
u_{i-1} \\
u_{i} \\
u_{i+1}
\end{array}\right]+\frac{1}{6}\left[\begin{array}{lll}
2 & 1 & 0 \\
1 & 4 & 1 \\
0 & 1 & 2
\end{array}\right]\left[\begin{array}{l}
\dot{u}_{i-1} \\
\dot{u}_{i} \\
\dot{u}_{i+1}
\end{array}\right]-\frac{B}{2 l^{(e)}}\left[\begin{array}{rrr}
-1 & 1 & 0 \\
-1 & 0 & 1 \\
0 & -1 & 1
\end{array}\right]\left[\begin{array}{l}
u_{i-1} \\
u_{i} \\
u_{i+1}
\end{array}\right]+\frac{Y}{6}\left[\begin{array}{lll}
2 & 1 & 0 \\
1 & 4 & 1 \\
0 & 1 & 2
\end{array}\right]\left[\begin{array}{l}
u_{i-1} \\
u_{i} \\
u_{i+1}
\end{array}\right]=\frac{P}{2}\left[\begin{array}{l}
1 \\
2 \\
1
\end{array}\right]
$$

Now put row corresponding to the node $i$ to zero, from equation (21) the difference schemes with $l^{(e)}=h$ is:

$$
\frac{1}{h^{2}}\left[-u_{i-1}+2 u_{i}-u_{i+1}\right]+\frac{1}{6}\left[\dot{u}_{i-1}+4 \dot{u}_{i}+\dot{u}_{i+1}\right]-\frac{B}{2 h}\left[-u_{i-1}+u_{i+1}\right]+\frac{Y}{6}\left[u_{i-1}+4 u_{i}+u_{i+1}\right]=P(22)
$$

Applying the trapezoidal rule, following system of equations in Crank - Nicholson method are obtained:

$$
A_{1} u_{i-1}^{n+1}+A_{2} u_{i}^{n+1}+A_{3} u_{i+1}^{n+1}=A_{4} u_{i-1}^{n}+A_{5} u_{i}^{n}+A_{6} u_{i+1}^{n}+12 P k
$$

Now from equations (13) and (14), following equations are obtained:

$$
\begin{aligned}
& B_{1} \theta_{i-1}^{n+1}+B_{2} \theta_{i}^{n+1}+B_{3} \theta_{i+1}^{n+1}=B_{4} \theta_{i-1}^{n}+B_{5} \theta_{i}^{n}+B_{6} \theta_{i+1}^{n}+12 Q k \\
& D_{1} C_{i-1}^{n+1}+D_{2} C_{i}^{n+1}+D_{3} C_{i+1}^{n+1}=D_{4} C_{i-1}^{n}+D_{5} C_{i}^{n}+D_{6} C_{i+1}^{n}
\end{aligned}
$$

Where $A_{1}=2+Y k+3 B r h-6 r, A_{2}=8+4 Y r h+12 r, A_{3}=2+Y k-3 B r h-6 r$,

$$
\begin{aligned}
& A_{4}=2-Y k-3 B r h+6 r, A_{5}=8-4 Y r h-12 r, A_{6}=2-Y k+3 B r h+6 r, \\
& B_{1}=2(\operatorname{Pr})+3 B r h(\operatorname{Pr})-S(\operatorname{Pr}) k-6 Z r, B_{2}=8(\operatorname{Pr})-4 S(\operatorname{Pr}) k+12 Z r, \\
& B_{3}=2(\operatorname{Pr})-3 B r h(\operatorname{Pr})-S(\operatorname{Pr}) k-6 Z r, B_{4}=2(\operatorname{Pr})-3 B r h(\operatorname{Pr})+S(\operatorname{Pr}) k+6 Z r, \\
& B_{5}=8(\operatorname{Pr})+4 S(\operatorname{Pr}) r h-6 Z r, B_{6}=2(\operatorname{Pr})+3 B r h(\operatorname{Pr})+S(\operatorname{Pr}) k+6 Z r, \\
& D_{1}=2(S c)+3 B r h(S c)+k_{r}(S c) k-6 r, D_{2}=8(S c)+4 k_{r}(S c) r h+12 r, \\
& D_{3}=2(S c)-3 B r h(S c)+k_{r}(S c) k-6 r, D_{4}=2(S c)-3 B r h(S c)-k_{r}(S c) k+6 r, \\
& D_{5}=8(S c)-4 k_{r}(S c) r h-12 r, D_{6}=2(S c)+3 B r h(S c)-k_{r}(S c) k+6 r, \\
& P^{*}=12 P h k=12 h k(G r) \theta_{i}^{j}+12 h k(G c) C_{i}^{j}, Q^{*}=12 k Q=12 k(\operatorname{Pr})(E c)\left(\frac{\partial u_{i}^{j}}{\partial y}\right)^{2}, Z=1+\frac{4}{3 R}
\end{aligned}
$$

Here $r=\frac{k}{h^{2}}$ and $h, k$ are mesh sizes along $y$-direction and time - direction respectively. Index $i$ refers to space and $j$ refers to the time. In the equations (23), (24) and (25) taking $i=1(1) n$ and using boundary conditions (15), then the following system of equations are obtained:

$A_{i} X_{i}=B_{i} ; i=1(1) n$

Where $A_{i}^{\prime}$ s are matrices of order $n$ and $X_{i}, B_{i}^{\prime}$ s are column matrices having $n$-components. The solutions of above system of equations are obtained by using Thomas algorithm for velocity, temperature and concentration. Also, numerical solutions for these equations are obtained by $C$-programme. In order to prove the convergence and stability of Galerkin finite element method, the same $C$-programme was run with smaller values of $h$ and $k$ and no significant change was observed in the values of $u, \theta$ and $C$. Hence the Galerkin finite element method is stable and convergent. 


\section{Results and Discussion:}

The formulation of the effects of chemical reaction, heat source and thermal radiation on MHD convective flow and mass transfer of an incompressible, viscous fluid along a semi - infinite vertical porous moving plate in a porous medium has been performed in the preceding sections. This enables us to carry out the numerical calculations for the distribution of the velocity, temperature and concentration across the boundary layer for various values of the parameters. In the present study we chosen $A=0.5, t=1.0, n=0.1, U_{p}=0.5$ and $\varepsilon=0.02$ and while $G r, G c, \operatorname{Pr}, S c, M, K, S, E c, R$ and $k_{r}$ are varied over a range, which are listed in the figure legends.

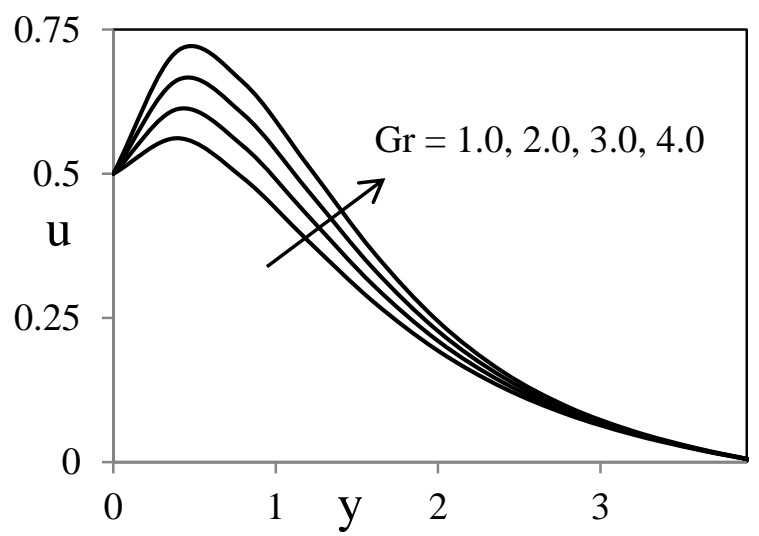

Figure 2. Velocity profiles for different values of thermal Grashof number $G r$

For the case of differentvalues of thermal Grashof number $G r$, the velocity profiles in the boundary layer are shown in figure (2). As expected, it is observed that an increase in $G r$ leads to decrease in the values ofvelocity due to enhancement in buoyancy force. Here the positive values of $G r$ correspond tocooling of the surface. In addition the curve show that the peak values of the velocitydecreases rapidly near the wall of the porous plate as Grashof number increases and thendecays to the free stream velocity. Figure (3) presents typical velocity profiles in the boundary layer for various values of the solutal Grashof number $G c$, while all other parameters are kept at some fixed values. The velocity distribution attains a distinctive maximum value in the vicinity of the plate surface and then decrease properly to approach the free stream value. As expected, the fluid velocity increases and the peak value more distinctive due to increase in the concentration buoyancy effects represented by $G c$. This is evident in the increase in the value of $u$ as increases $G c$ in figure (3).

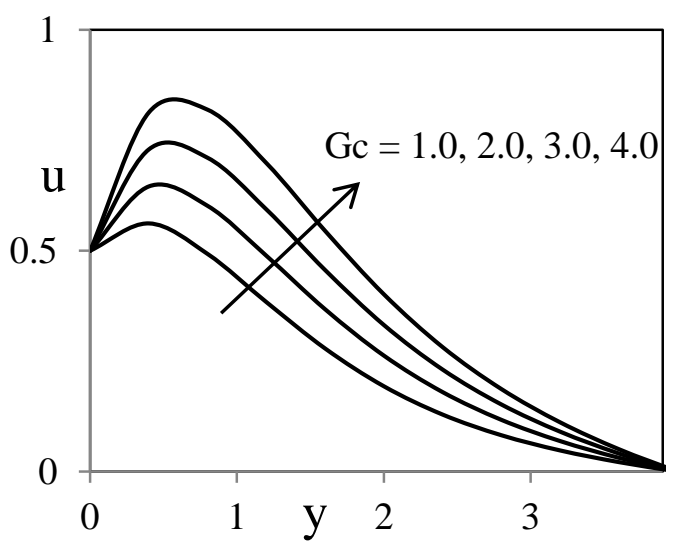

Figure 3. Velocity profiles for different values of solutal Grashof number $G c$ 


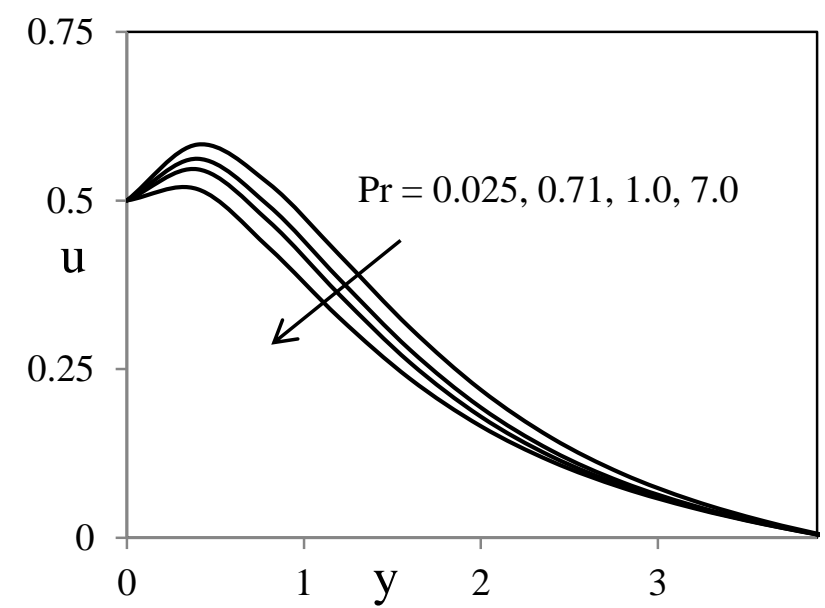

Figure 4. Velocity profiles for different values of Prandtl number $\mathrm{Pr}$

Figure (4) and (5) illustrate the velocity and temperature profiles for different values of Prandtl number Pr . The numerical results show that the effectof increasing values of Prandtl number result in an decreasing velocity. From figure (5) asexpected, the numerical results show that an increase in the Prandtl number results a decrease of the thermal boundary layer and in general lower average temperature within the boundarylayer. The reason is that smaller values of $\operatorname{Pr}$ are equivalent to increase in the thermalconductivity of the fluid and therefore heat is able to diffuse away from the heated surfacemore rapidly for higher values of $\operatorname{Pr}$. Hence in the case of smaller Prandtl number the thermalboundary layer is thicker and the rate of heat transfer is reduced.

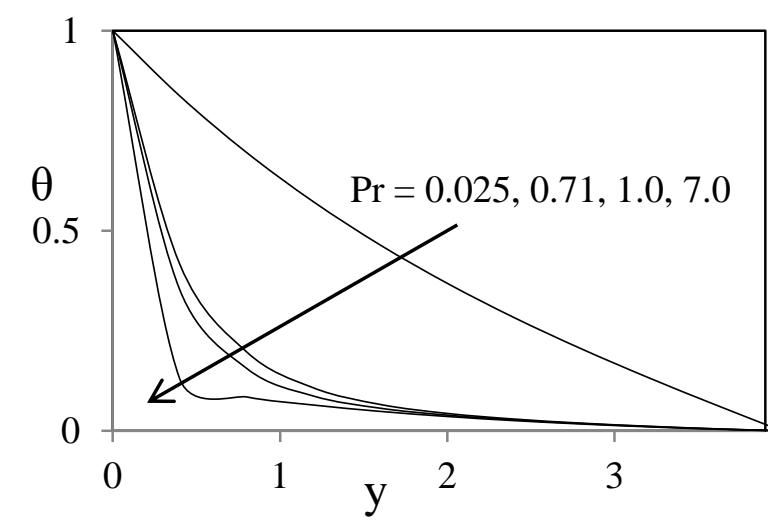

Figure 5. Temperature profiles for different values of $\mathrm{Pr}$

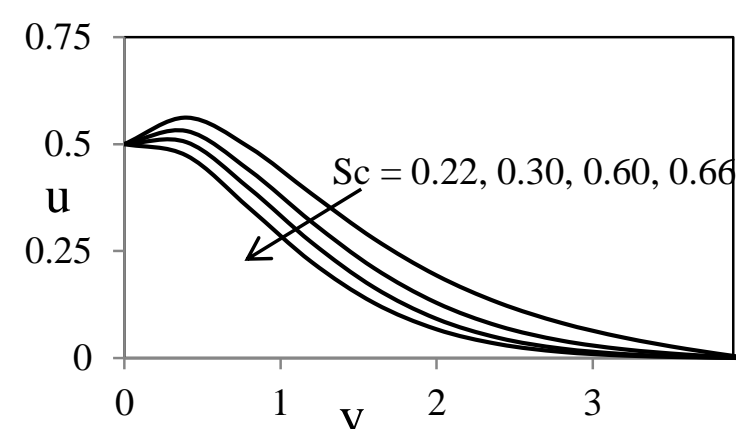

Figure 6. Velocity profiles for different values of Schmidt number $S c$

Figure (6) and (7) display the effects of Schmidt number $S c$ on velocity and concentration profiles respectively. As the Schmidt number increases the concentration decreases. This causes the concentration buoyancy effects to decreases yielding a reduction in the fluid velocity. The reductions and the velocity and concentration profiles are accompanied by simultaneous reductions in the velocity and concentration boundary layers. These behaviors are clearly shown in figures (6) and (7). 


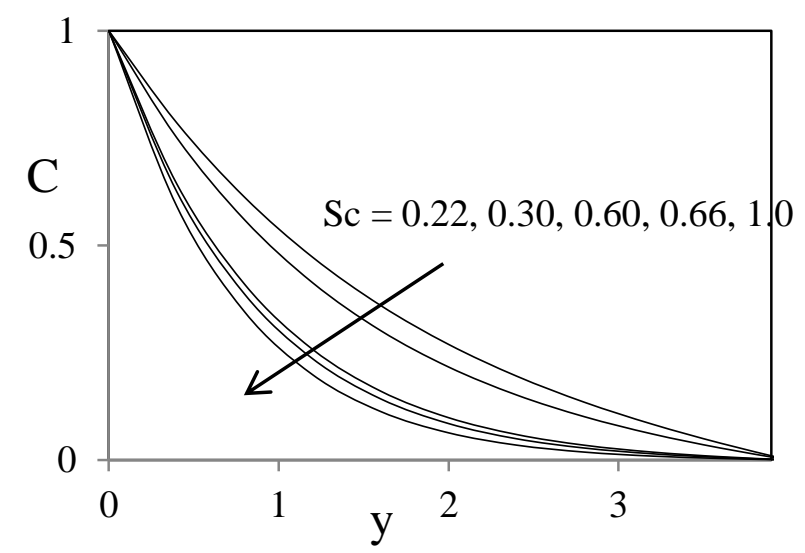

Figure 7. Concentration profiles for different values of Schmidt number $S c$

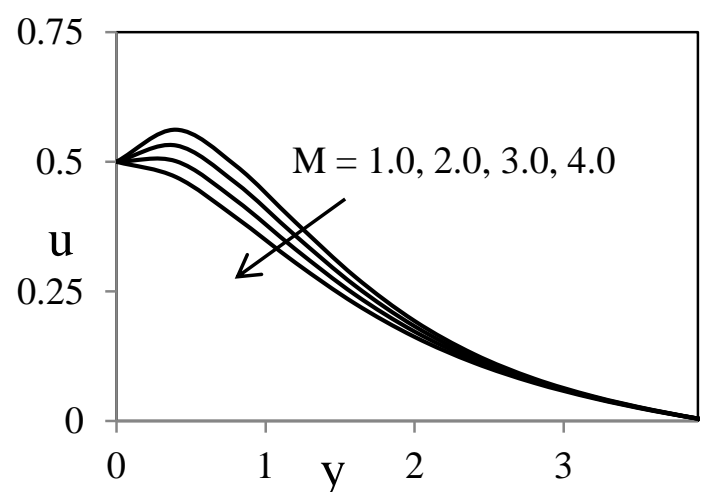

Figure 8. Velocity profiles for different values of Hartmann number $M$

The effect of Hartmann number on velocity profiles in the boundary layer is depicted in figure (8). From this figure it is seen that the velocity starts from minimum value at the surface and increase till it attains the peak value and then starts decreasing until it reaches to the minimum value at the end of the boundary layer for all the values of Hartmann number. It is interesting to note that the effect of magnetic field is to decrease the value of the velocity profiles throughout the boundary layer. The effect of magnetic field is more prominent at the point of peak value i.e. the peak value drastically decreases with increases in the value of magnetic field, because the presence of magnetic field in an electrically conducting fluid introduce a force called the Lorentz force, which acts against the flow if the magnetic field is applied in the normal direction, as in the present problem. This type of resisting force slows down the fluid velocity as shown in this figure. Figure (9) Shows the velocity profiles for different values of the permeability parameter $K$, clearly as $K$ increases the peak values of the velocity tends to increase. The effect of increasing the value of the heat source parameter $S$ is to decrease the boundary layer as shown in figure (10), which is as expected due to the fact that when heat is absorbed the buoyancy force decreases which retards the flow rate and thereby giving rise to decrease in the velocity profiles.

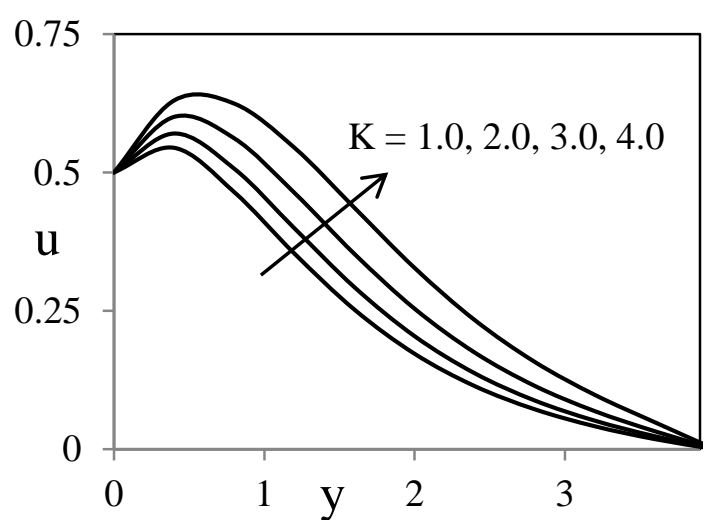

Figure 9. Velocity profiles for different values of Permeability parameter $K$ 




Figure 10. Velocity profiles for different values of Heat source parameter $S$

Figure (11) has been plotted to depict the variation of temperature profiles against $y$ for different values of heat source parameter $S$ by fixing other physical parameters. From this graph we observe that temperature $\theta$ decrease with increase in the heat source parameter $S$ because when heat is absorbed, the buoyancy force decreases the temperature profiles. Figures (12) and (13) display the effects of the chemical reaction parameter $\left(k_{r}\right)$ on the velocity and concentration profiles, respectively. As expected, the presence of the chemical reaction significantly affects the concentration profiles as well as the velocity profiles. It should be mentioned that the studied case is for a destructive chemical reaction $\left(k_{r}\right)$. In fact, as chemical reaction $\left(k_{r}\right)$ increases, the considerable reduction in the velocity profiles is predicted, and the presence of the peak indicates that the maximum value of the velocity occurs in the body of the fluid close to the surface but not at the surface. Also, with an increase in the chemical reaction parameter, the concentration decreases. It is evident that the increase in the chemical reaction $\left(k_{r}\right)$ significantly alters the concentration boundary layer thickness but does not alter the momentum boundary layers.

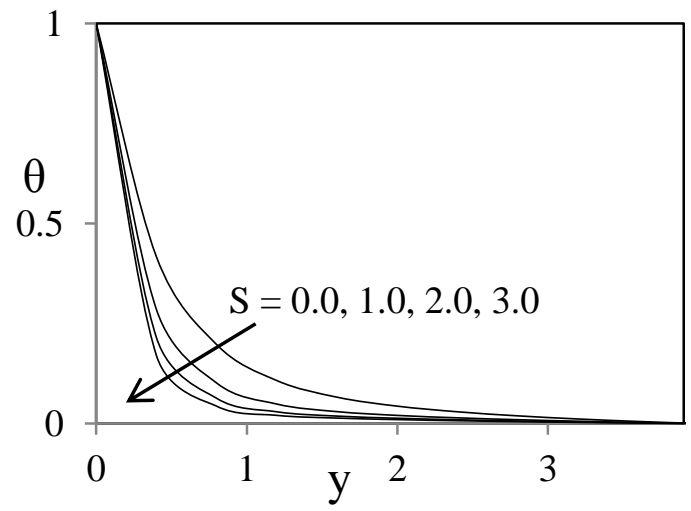

Figure 11. Temperature profiles for different values of Heat source parameter $S$

Figure (14) illustrates the variation of velocity distribution across the boundary layer for several values of plate $U_{p}$ moving velocity in the direction of the fluid flow. Although we have different initial plate moving velocities, the velocity decrease to the constant values for given material parameters. The influence of the viscous dissipation parameter i.e., the Eckert number $(E c)$ on the velocity and temperature are shown in figures $(15)$ and (16) respectively. The Eckert number $(E c)$ expresses the relationship between the kinetic energy in the flow and the enthalpy. It embodies the conversion of kinetic energy into internal energy by work done against the viscous fluid stresses. Greater viscous dissipative heat causes a rise in the temperature as well as the velocity. This behavior is evident from figures (15) and (16). 


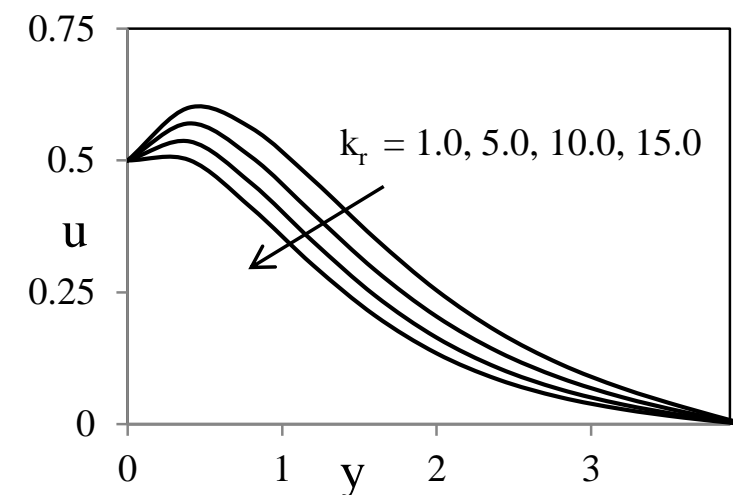

Figure 12. Velocity profiles for different values of Chemical reaction parameter $k_{r}$

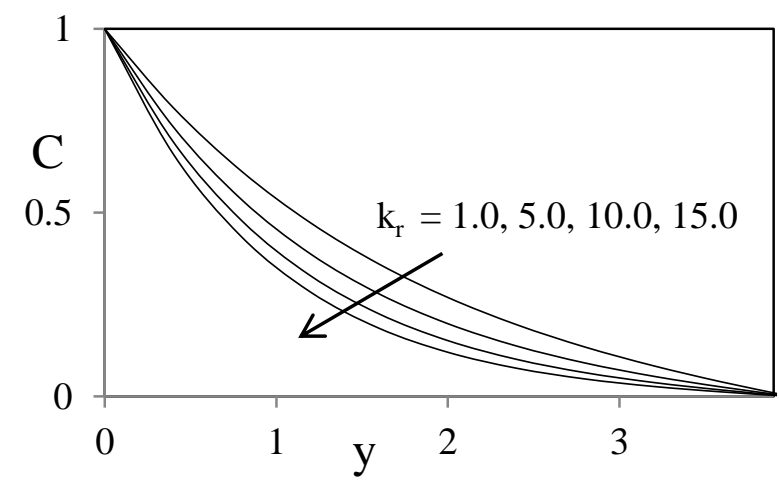

Figure 13. Concentration profiles for different values of Chemical reaction parameter $k_{r}$

Figures (17) and (18) illustrate the variation of velocity and temperature for various values of the radiation absorption parameter $(R)$. It is immediately apparent that velocity as well as temperature clearly decrease as $R$ rises from 1 to 3 . Velocity reaches a maximum in close proximity to the wall and then falls gradually to zero at the edge of the boundary layer. Inspection of figure (18) shows that for a small value of $R(R<2)$ temperature profile continuously decreases from the wall, while for higher values of $R$ it increases attaining a maximum near the plate boundary and then decreases. As such there is a noticeable temperature overshoot with $R>1$ since considerable thermal energy is imparted via the presence of a thermal radiation source to the fluid causing an elevation in temperatures near the wall.

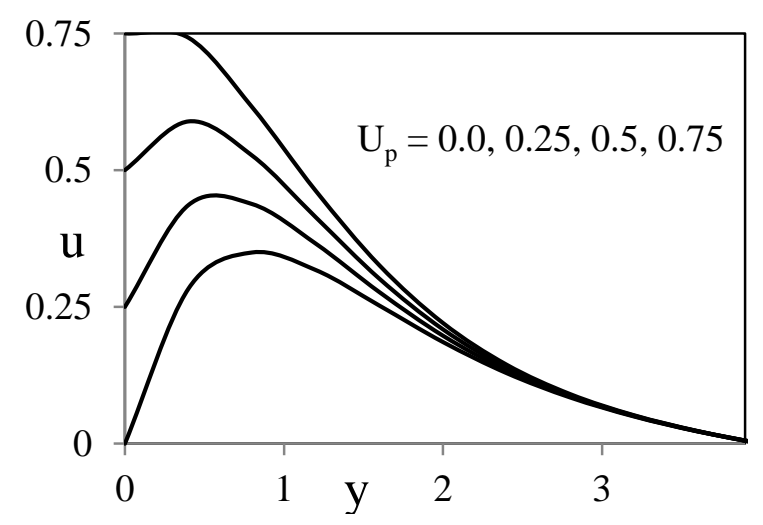

Figure 14. Velocity profiles for different values of Plate velocity $U_{p}$ 


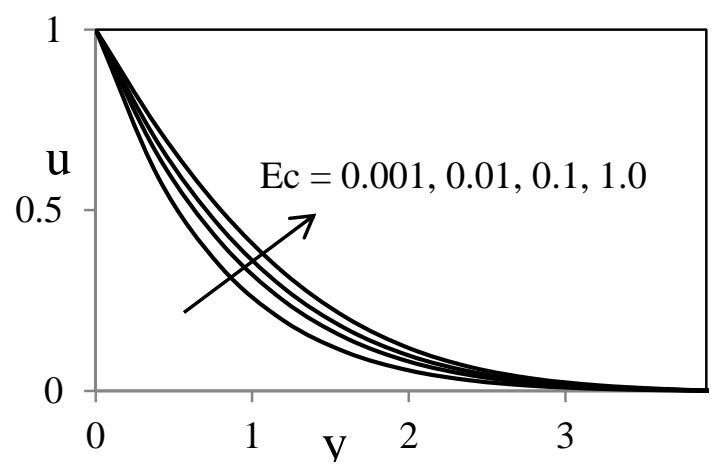

Figure 15. Velocity profiles for different values of Eckert number $E c$

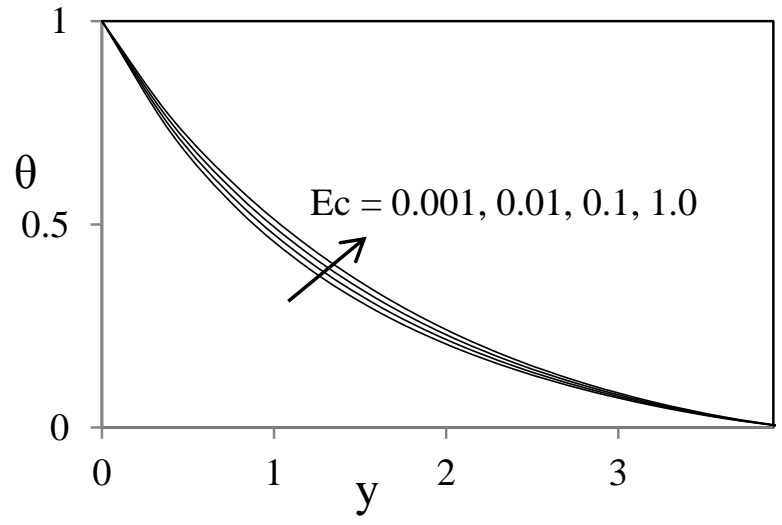

Figure 16. Temperature profiles for different values of Eckert number $E c$

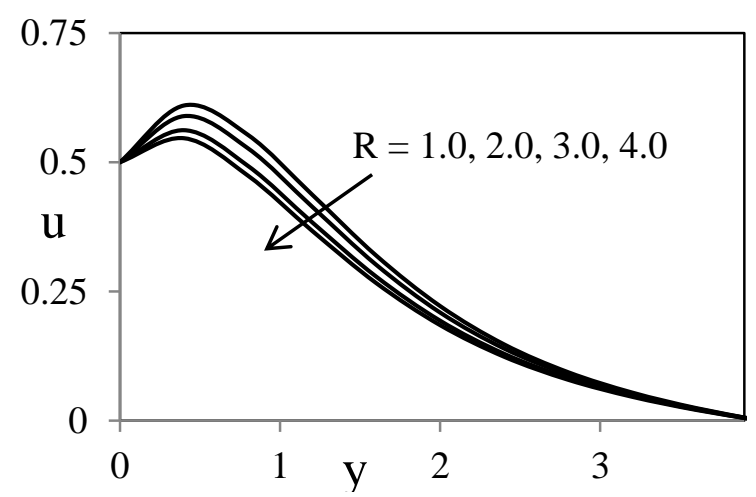

Figure 17. Velocity profiles for different values of Thermal radiation parameter $R$

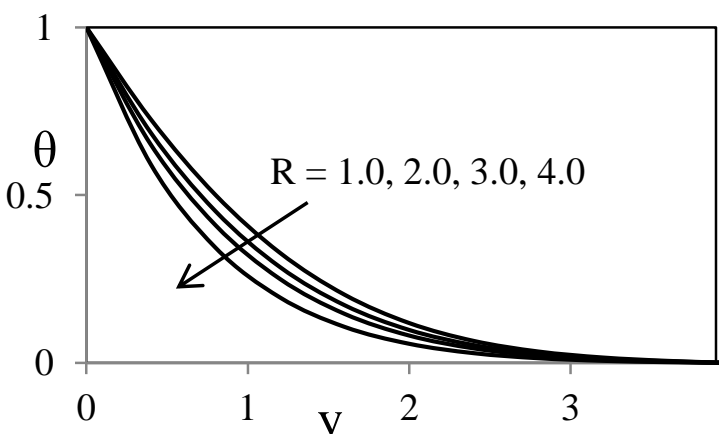

Figure 18. Temperature profiles for different values of Thermal radiation parameter $R$

Table - 1: Skin - friction results $(\tau)$ for the values of $G r, G c, \operatorname{Pr}, S c, M, K, \phi$ and $k_{r}$

\begin{tabular}{|l|l|l|l|l|l|l|l|l|l|l|}
\hline$G r$ & $G c$ & $\operatorname{Pr}$ & $S c$ & $M$ & $K$ & $S$ & $R$ & $E c$ & $k_{r}$ & $\tau$ \\
\hline
\end{tabular}


Thermal radiation effect on an unsteady MHD free convective chemically reacting viscous dissipative

\begin{tabular}{|l|l|l|l|l|l|l|l|l|l|l|}
\hline 1.0 & 1.0 & 0.71 & 0.22 & 1.0 & 1.0 & 1.0 & 1.0 & 0.001 & 1.0 & 2.2858 \\
\hline 2.0 & 1.0 & 0.71 & 0.22 & 1.0 & 1.0 & 1.0 & 1.0 & 0.001 & 1.0 & 2.3549 \\
\hline 1.0 & 2.0 & 0.71 & 0.22 & 1.0 & 1.0 & 1.0 & 1.0 & 0.001 & 1.0 & 2.5487 \\
\hline 1.0 & 1.0 & 7.00 & 0.22 & 1.0 & 1.0 & 1.0 & 1.0 & 0.001 & 1.0 & 2.1741 \\
\hline 1.0 & 1.0 & 0.71 & 0.30 & 1.0 & 1.0 & 1.0 & 1.0 & 0.001 & 1.0 & 2.2016 \\
\hline 1.0 & 1.0 & 0.71 & 0.22 & 2.0 & 1.0 & 1.0 & 1.0 & 0.001 & 1.0 & 2.1995 \\
\hline 1.0 & 1.0 & 0.71 & 0.22 & 1.0 & 2.0 & 1.0 & 1.0 & 0.001 & 1.0 & 2.3353 \\
\hline 1.0 & 1.0 & 0.71 & 0.22 & 1.0 & 1.0 & 2.0 & 1.0 & 0.001 & 1.0 & 2.2217 \\
\hline 1.0 & 1.0 & 0.71 & 0.22 & 1.0 & 1.0 & 1.0 & 2.0 & 0.001 & 1.0 & 2.1882 \\
\hline 1.0 & 1.0 & 0.71 & 0.22 & 1.0 & 1.0 & 1.0 & 1.0 & 0.100 & 1.0 & 2.2945 \\
\hline 1.0 & 1.0 & 0.71 & 0.22 & 1.0 & 1.0 & 1.0 & 1.0 & 0.001 & 2.0 & 2.1532 \\
\hline
\end{tabular}

Table - 2: Rate of heat transfer $(N u)$ values for different values of $\operatorname{Pr}$ and $\phi$

\begin{tabular}{|c|c|c|c|c|}
\hline $\operatorname{Pr}$ & $S$ & $R$ & $E c$ & $N u$ \\
\hline 0.71 & 1.0 & 1.0 & 0.001 & 0.5841 \\
\hline 7.00 & 1.0 & 1.0 & 0.001 & 0.5149 \\
\hline 0.71 & 2.0 & 1.0 & 0.001 & 0.5016 \\
\hline 0.71 & 1.0 & 2.0 & 0.001 & 0.5268 \\
\hline 0.71 & 1.0 & 1.0 & 0.100 & 0.5924 \\
\hline
\end{tabular}

Table - 3: Rate of mass transfer ( $S h$ ) values for different values of $S c$ and $k_{r}$

\begin{tabular}{|c|c|c|}
\hline$S c$ & $k_{r}$ & $S h$ \\
\hline 0.22 & 1.0 & 0.4492 \\
\hline 0.30 & 1.0 & 0.4212 \\
\hline 0.22 & 2.0 & 0.4110 \\
\hline
\end{tabular}

Table - 4: Comparison of present Skin - friction results $(\tau)$ with the Skin - friction results $\left(\tau^{*}\right)$ obtained by Kesavaiah et al. [5] for different values of $G r, G c, \operatorname{Pr}, S c, M, K, S$ and $k_{r}$

\begin{tabular}{|c|c|c|c|c|c|c|c|c|c|}
\hline$G r$ & $G c$ & $\operatorname{Pr}$ & $S c$ & $M$ & $K$ & $S$ & $k_{r}$ & $\tau$ & $\tau^{*}$ \\
\hline 1.0 & 1.0 & 0.71 & 0.22 & 1.0 & 1.0 & 1.0 & 1.0 & 2.2858 & 2.2847 \\
\hline 2.0 & 1.0 & 0.71 & 0.22 & 1.0 & 1.0 & 1.0 & 1.0 & 2.3549 & 2.3536 \\
\hline 1.0 & 2.0 & 0.71 & 0.22 & 1.0 & 1.0 & 1.0 & 1.0 & 2.5487 & 2.5462 \\
\hline 1.0 & 1.0 & 7.00 & 0.22 & 1.0 & 1.0 & 1.0 & 1.0 & 2.1741 & 2.1728 \\
\hline 1.0 & 1.0 & 0.71 & 0.30 & 1.0 & 1.0 & 1.0 & 1.0 & 2.2016 & 2.2011 \\
\hline 1.0 & 1.0 & 0.71 & 0.22 & 2.0 & 1.0 & 1.0 & 1.0 & 2.1995 & 2.1971 \\
\hline 1.0 & 1.0 & 0.71 & 0.22 & 1.0 & 2.0 & 1.0 & 1.0 & 2.3353 & 2.3339 \\
\hline 1.0 & 1.0 & 0.71 & 0.22 & 1.0 & 1.0 & 2.0 & 1.0 & 2.2217 & 2.2206 \\
\hline 1.0 & 1.0 & 0.71 & 0.22 & 1.0 & 1.0 & 1.0 & 2.0 & 2.1882 & 2.1855 \\
\hline
\end{tabular}

The profiles for skin - friction $(\tau)$ due to velocity under the effects of thermal Grashof number, solutal Grashof number, Prandtl number, Schmidt number, Hartmann number, Permeability parameter,Heat source parameter, Thermal radiation parameter and Chemical reaction parameter are presented in the table -1 respectively. We observe from this table -1 , the skin - friction $(\tau)$ rises under the effects of thermal Grashof number, solutal Grashof number, Eckert number and Permeability parameter. And falls under the effects of Prandtl number, Schmidt number, Hartmann number, Heat source parameter, Thermal radiation parameter and Chemical reaction parameter. The profiles for Nusselt number $(\mathrm{Nu})$ due to temperature profile under the effect of Prandtl number, Eckert number, Thermal radiation parameter and Heat source parameter are presented in the table -2 . From this table we observe that, the Nusselt number due to temperature profiles falls under the effects of Prandtl number, Thermal radiation parameter and Heat source parameter and rises under the effect of Eckert number. The profiles for Sherwood number $(S h)$ due to concentration profiles under the effect of Schmidt number and Chemical reaction parameter are presented in the table -3 . We see from this table the Sherwood number due to concentration profiles decreases under the effects of Schmidt number and Chemical reaction parameter. In order to ascertain the accuracy of the numerical results, the present results are compared with the 
previous results of Kesavaiah et al. [5] for $G r=G c=1.0, \operatorname{Pr}=0.71, S c=0.22, M=1.0, K=1.0, S=1.0$ and $k_{r}=1.0$ in table -4 . They are found to be in an excellent agreement.

\section{Conclusions:}

The governing equations for heat and mass transfer by laminar flow of a Newtonian, viscous, electrically conducting chemically reacting viscous dissipative fluid on a continuously vertical permeable surface in the presence of a heat source, a first - order homogeneous chemical reaction and the mass flux are reported. The resulting partial differential equations were transformed into a set of partial differential equations solved by using finite element method. Numerical evaluations of the numerical results were performed and graphical results were obtained to illustrate the details of the flow and heat and mass transfer characteristics and their dependence on some physical parameters.

1. An increasing Hartmann number, Prandtl number, Schmidt number, Heat source parameter, Thermal radiation parameter and Chemical reaction parameter decreases the velocity of the flow field at all points.

2. An increasing thermal Grashof number, solutal Grashof number, Permeability parameter, Eckert number and Plate velocity is to increase the velocity of the flow field at all points.

3. A growing Prandtl number, Thermal radiation parameter and Heat absorption parameter decreases temperature of the flow field at all points.

4. The Schmidt number and Chemical reaction parameter decreases the concentration of the flow field at all points.

5. An increasing Hartmann number, Prandtl number, Schmidt number, Heat source parameter, Thermal radiation parameter and Chemical reaction parameter decreases the skin - friction coefficient $(\tau)$.

6. An increasing thermal Grashof number, solutal Grashof number, Permeability parameter, Eckert number and Plate velocity is to increase skin - friction coefficient $(\tau)$.

7. A growing Prandtl number, Thermal radiation parameter and Heat absorption parameter decreases Nusselt number of the flow field at all points.

8. The Schmidt number and Chemical reaction parameter decreases the Sherwood number of the flow field at all points.

9. On comparing the skin - friction $(\tau)$ results with the skin - friction $\left(\tau^{*}\right)$ results of Kesavaiah et al. [5] it can be seen that they agree very well.

[1] Abdur Sattar, Md. and Kh. Abdul Maleque, 2005. The Effects of Variable properties and Hall current on steady MHD laminar convective fluid flow due to a porous rotating disk, Int. Journal of Heat and Mass Transfer, Vol. 48, pp. 4460 - 4466.

[2] Anjali Devi, S. P. and J. Wilfred Samuel Raj,2011. Thermo diffusion effects on unsteady hydromagnetic free convection flow with heat and mass transfer past a moving vertical plate with time dependent suction and heat source in a slip flow regime,Int. J. of Appl. Math. and Mech., Vol. 7, No. 21, pp. $20-51$.

[3] Dulal Pal and Babulal Talukdar, 2010. Perturbation analysis of unsteady magnetohydro dynamic convective heat and mass transfer in a boundary layer slip flow past a vertical permeable plate with thermal radiation and chemical reaction, Communications in Nonlinear Science and Numerical Simulation,pp.1813-1830.

[4] Ibrahim, F. S., A. M. Elaiw and A. A. Bakr, 2008. Effect of the chemical reaction and radiation absorption on the unsteady MHD free convection flow past a semi - infinite vertical permeable moving plate with heat source and suction, Communications Nonlinear Science Numerical Simulation, Vol. 13, No. 6, pp. 1056 - 1066.

[5] Kesavaiah, D., Ch. P. V. Satyanarayana and S. Venkataramana, 2011. Effects of the chemical reaction and radiation absorption on an unsteady MHD convective heat and mass transfer flow past a semi - infinite vertical permeable moving plate embedded in a porous medium with heat source and suction,Int. J. of Appl. Math. and Mech., Vol. 7, No. 1, pp. 52 - 69.

[6] Loganathan, P., T, Kulandaivel and R. Muthucumaraswamy, 2008. First order chemical reaction on moving semi - infinite vertical plate in the presence of optically thin gray gas,Int. J. of Appl. Math. and Mech., Vol. 4, No. 5, pp. 26 - 41.

[7] Mansour, M. A., N. F. El - Anssary and A. M. Aly, 2008. Effect of chemical reaction and viscous dissipation on MHD natural convection flows saturated in porous media with suction or injection, Int. J. of Appl. Math. and Mech., Vol. 4, No. 2, pp. 60 - 76.

[8] Mohamad, 2009. Double diffusive convection - radiation interaction on unsteady MHD flow over a vertical moving porous plate with heat generation and Soret effect was studied, Applied Mathematical Sciences, Vol. 13, pp. $629-651$.

[9] Muthucumaraswamy, R. and A. Vijayalakshmi, 2008. Effects of heat and mass transfer on flow past an Oscillating vertical plate with variable temperature, Int. J. of Appl. Math. and Mech., Vol. 4, No. 1, pp. $59-65$.

[10] Muthucumaraswamy, R. and S. Meenakshisundaram, 2006. Theoretical study of chemical reaction effects on vertical oscillating plate with variable temperature, Theoret. Appl. Mech., Vol. 33, No. 3, pp. 245 - 257.

[11] Muthuraj, R. and S. Srinivas, 2010. Fully developed MHD flow of a micropolar and viscous fluid in a vertical porous space using HAM, Int. J. Appl. Mathematics and Mechanics, Vol. 6, No. 11, pp. 79 - 97.

[12] Ogulu, A., A. R. C. Amakiri. and I. U. Mbeledogu, 2007. Unsteady MHD free convective flow of a compressible fluid past a moving vertical plate in the presence of radiative heat transfer, Int. Journal of Heat and Mass Transfer, Vol. 50 , pp. 1668 - 1674.

[13] Patil, P. M. and P. S. Kulkarni, 2008. Effects of chemical reaction on free convective flow of a polar fluid through a porous medium in the presence of internal heat generation, Int. J. Therm. Sci.,Vol. 4, pp. 1043 - 1054. 
[14] Ramachandra Prasad, V. and N. Bhaskar Reddy, 2008. Radiation effects on an unsteady MHD convective heat and mass transfer flow past a semi - infinite vertical permeable moving plate embedded in a porous medium, Journals of Energy Heat and mass transfer,Vol. 30, pp.57-68.

[15] Ramana Reddy, G. V., Ch. V. Ramana Murthy and N. Bhaskar Reddy, 2010. Mass transfer and radiation effects of unsteady MHD free convective fluid flow embedded in porous medium with heat generation/absorption, Journal of Applied Mathematics and Fluid Mechanics, Vol. 2, No. 1, pp. $85-98$.

[16] Samad, Sk. A., G. C. Layek and S. Mukhopadhyaya, 2005. MHD boundary layer flow over a heated stretching sheet with variable Viscosity, Int. Journal of Heat and Mass Transfer, Vol. 48, pp. $4460-4466$.

[17] Satyanarayana, P. V. and S. Venkataramana, 2007. Hall current effect on magnetohydro dynamics freeconvection flow past a semi - infinite vertical porous plate with mass transfer. S.V.University, Ph.D thesis.

[18] Seddeek, M. A., A. A. Darwish and M. S. Abdelmeguid, 2007. Effects of chemical reaction and variable viscosity on hydromagnetic mixed convection heat and mass transfer for Hiemenz flow through porous media with radiation, Communications in Nonlinear Science and Numerical Simulation, Vol. 12, No. 2, pp. 195 - 213.

[19] Sharma, P. R. and G. Singh, 2008.Unsteady MHD free convective flow and heat transfer along a vertical porous plate with variable suction and internal heat generation,Int. J. of Appl. Math. and Mech., Vol. 4, No. 5, pp. $1-8$.

[20] Sudheer Babu, M. and P. V. Satyanarayana, 2009. Effects of the chemical reaction and radiation absorption on free convection flow through porous medium with variable suction in the presence of uniform magnetic field, J. P. Journal of Heat and mass transfer, Vol. 3, pp. $219-234$.

[21] Venkateswalu, S., K. V. Suryanarayana Rao and B. Rambupal Reddy, 2011. Finite difference analysis on convective heat transfer flow through a porous medium in a vertical channel with magnetic field,Int. J. of Appl. Math. and Mech., Vol. 7, No. 7, pp. $74-94$.

[22] Zueco, J., 2007. Network simulation method applied to radiation and viscous dissipationeffects on MHD unsteady free convection over vertical porous plate,Applied Mathematical Modelling, Vol. 31,No. 9, pp. 2019 - 2033

[23] Cogley, A. C., Vincenty, W. E. and Gilles, S. E., (1968). Differential approximation for radiation in a non-gray gas near equilibrium, A. I. A. A. J.,Vol. 6, pp. 551-553.

[24] Abdus Sattar, M. D., Kalim, M. D. and Hamid, (1996). Unsteady free convection interaction with thermal radiation in a boundary layer flow past a vertical porous plate, J. Math. Phys. Sci., Vol. 30, pp. 25-37.

[25] Vajravelu, K., (1994). Flow and heat transfer in a saturated porous medium, ZAMM, Vol. 74, pp. 605-614.

[26] Hossain, M. A. and Takhar, H. S., (1996). Radiation effect on mixed convection along a vertical plate with uniform surface temperature, Heat Mass Transfer, Vol. 31, pp. 243-248.

[27] Raptis, A., (1998). Radiation and free convection flow through a porous medium, Int. Commun. Heat Mass Transfer,Vol. 25, pp. $289-295$.

[28] Makinde, O. D., (2005). Free convection flow with thermal radiation and mass transfer past a moving vertical porous plate, Int. Commun. Heat Mass Transfer, Vol. 32, pp. 1411-1419.

[29] Ibrahim, F. S., Elaiw, A. M. and Bakr, A. A., (2008). Effect of the chemical reaction and radiation absorption on unsteady MHD mixed convection flow past a semiinfinite vertical permeable moving plate with heat source and suction, Commun. Nonlinear Sci. Numer. Simul., Vol. 13, pp. 1056-1066.

[30] Bakr, A. A., (2011). Effects of chemical reaction on MHD free convection and mass transfer flow of a micropolar fluid with oscillatory plate velocity and constant heat source in a rotating frame of reference, Commun. Nonlinear Sci. Numer. Simul., Vol. 16, pp. 698-710.

[31] Brewster, M. Q., Thermal radiative transfer \& properties, John Wiley \& Sons (1992). 